\title{
Brittle stars (Echinodermata: Ophiuroidea) in the German Bight (North Sea) - species diversity during the past 130 years
}

\author{
Karin Boos ${ }^{\dagger}$ and Heinz-Dieter Franke* \\ *Biologische Anstalt Helgoland, Foundation Alfred Wegener Institute for Polar and Marine Research, PO Box 180, \\ 27483 Helgoland, Germany. ${ }^{\dagger}$ Corresponding author, e-mail: kboos@awi-bremerhaven.de
}

In this study, an overview of the current species composition of ophiuroids off Helgoland, German Bight (North Sea) is given. In addition, abundance and distribution of ophiuroids on different types of soft bottom sediments taken by van Veen grab samples around Helgoland were recorded. The literature was reviewed in order to outline the diversity of ophiuroid species over the past 130 years in the inner German Bight. In the historical literature, quantitative references often apply to verbal descriptions and thus make comparisons to modern data and assessment of possible changes in abundance rather subjective.

In total, six ophiuroid species were identified off Helgoland: Acrocnida brachiata, Amphiura filiformis, Amphipholis squamata, Ophiothrix fragilis, Ophiura albida and Ophiura ophiura. The species found in this study had also been reported by previous investigators and are regarded as 'common' species in the German Bight. Occasional findings in this area refer to Amphiura chiajei, Ophiopholis aculeata, Ophiocten affinis and Ophiura sarsi, which are regarded here as 'rare' species. These findings, however, do not indicate sustainable changes in the species diversity over time.

Apart from Acrocnida brachiata, a newcomer in the 1970s, findings of the common species mentioned above can be dated back to 1875 . Therefore, a fairly stable composition of brittle stars is represented in the inner German Bight during the past 130 years.

\section{INTRODUCTION}

Species spectrum and abundances in the North Sea apparently have undergone profound and rapid changes in recent years. The reasons for this may be diverse: fishery, eutrophication, introduction of non-indigenous species, climate change (Reise et al., 1999; Franke \& Gutow, 2004; Reichert \& Buchholz, 2006). The echinoderm fauna of the German Bight has been described along with various studies dealing with the species diversity of different faunal associations (Stripp, 1969; Ziegelmeier, 1978; Salzwedel et al., 1985; Thatje \& Gerdes, 1997). Hagmeier (1925) was the first to present quantitative data on the faunal composition of benthic communities in the German Bight, while earlier investigations in the central and southern North Sea including the German Bight focused on qualitative data (Möbius \& Bütschli, 1875; Meissner \& Collin, 1894; Süßbach \& Breckner, 1911).

In a comprehensive investigation on the echinoderm fauna of the central North Sea, Ursin (1960) presented data from the Danish grab surveys (1932-1955) and compared them with the results of a number of previous studies from adjacent coastal areas, including the German Bight. Gerdes (1977) gave a summary of the distribution of echinoderms in the German Bight comparing his results with those of previous investigations as well. Both authors suggested little change in species composition over time. The objective of the present study was to give an overview of the current species composition of ophiuroids off Helgoland on different types of sediment as well as of the German Bight and to assess changes in the diversity of ophiuroid species over time.

\section{MATERIALS AND METHODS}

Processing of samples and literature data

From June to August 2003 van Veen grab sampling $\left(0.1 \mathrm{~m}^{2}\right)$ was performed at eight different stations on soft

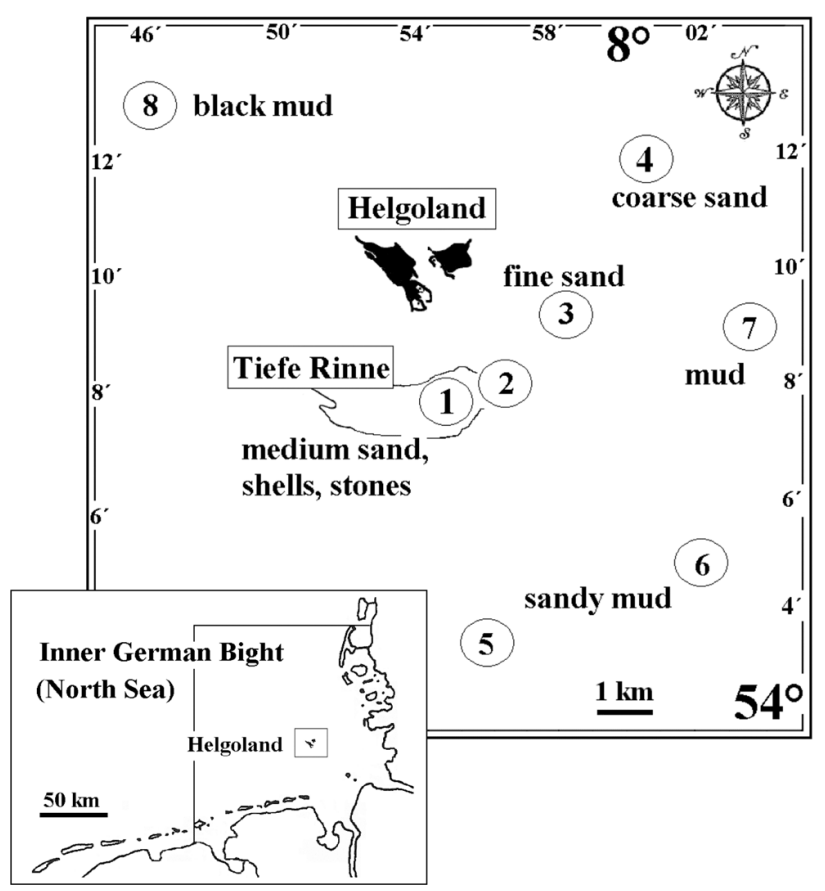

Figure 1. Sampling stations (1-8) around Helgoland and the location in the inner German Bight (North Sea) (area of investigation); modified after Benoit, 1998. 

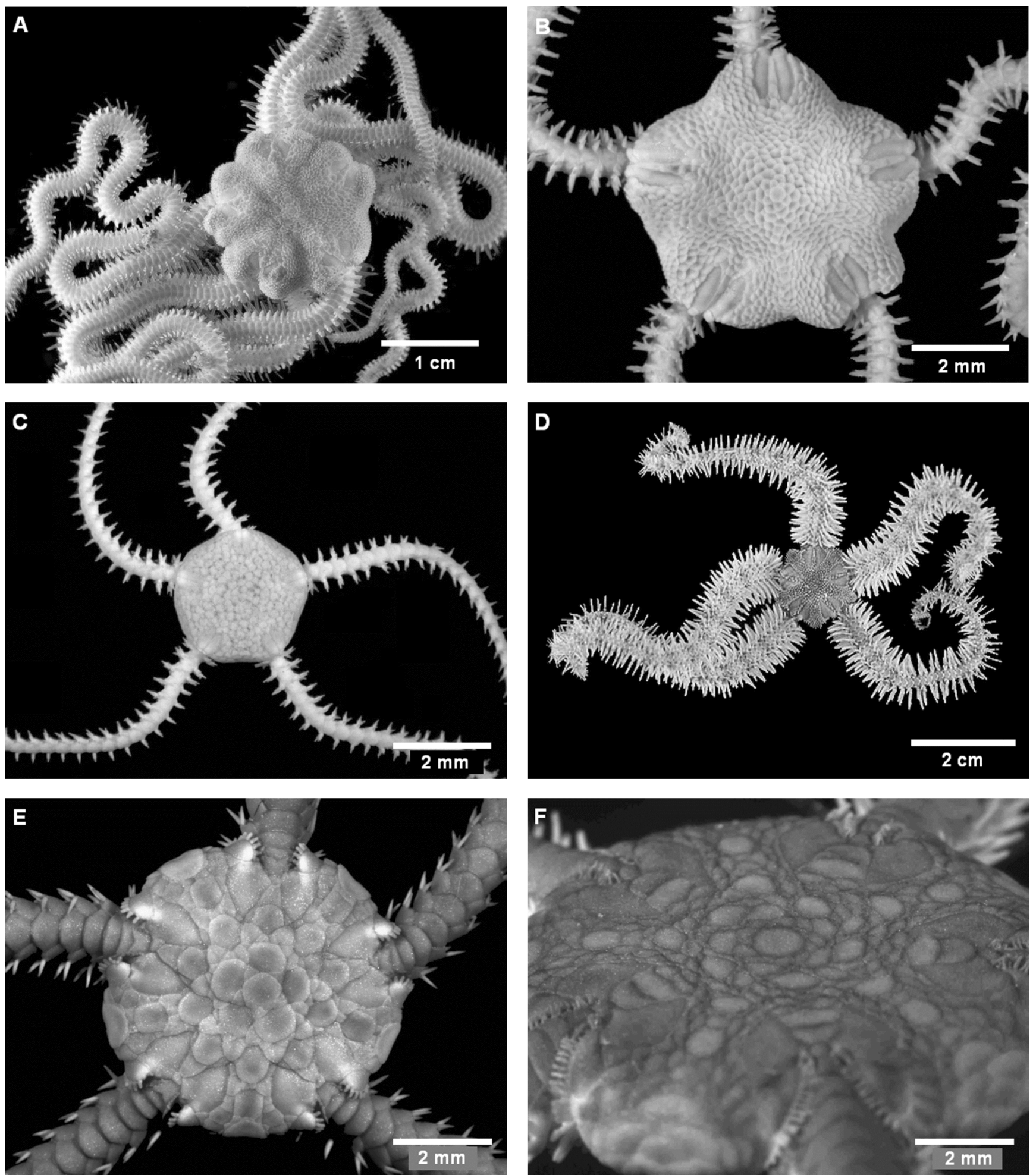

Figure 2. (A-F) Ophiuroid species found off Helgoland, German Bight (North Sea) in dorsal view. (A) Acrocnida brachiata; (B) Amphiura filiformis; (C) Amphipholis squamata; (D) Ophiothrix fragilis; (E) Ophiura albida; and (F) Ophiura ophiura.

bottom sediments in the vicinity of the Island of Helgoland, German Bight (North Sea) (Figure 1). Additional dredging was performed for complementation of the current spectrum of species. Due to lack of quantitative dredge sampling, however, information on abundances was estimated in categories 'low, medium and high'.
The stations ranged within a radius of approximately 5 to 6 nautical miles around Helgoland and were located in the area of $54^{\circ} 13^{\prime}-54^{\circ} 03^{\prime} \mathrm{N} 7^{\circ} 45^{\prime}-8^{\circ} 03^{\prime} \mathrm{E}$. The grab samples covered a total of $0.3 \mathrm{~m}^{2}$ per station. One litre of sediment from each grab sample was retained for sedimentary analysis. On board the research vessel, the samples were passed through a 1-mm sieve and the retained fauna 
Table 1. Characteristics of stations (position, depth, sediment) and abundance of ophiuroids per station in grab samples (total area of $0.3 \mathrm{~m}^{2}$ each); categories in parentheses (low, medium, high) refer to abundances estimated from dregde samples.

\begin{tabular}{|c|c|c|c|c|c|c|c|c|}
\hline Stations & 1 & 2 & 3 & 4 & 5 & 6 & 7 & 8 \\
\hline \multirow[t]{2}{*}{ Position } & $54^{\circ} 08^{\prime} \mathrm{N} /$ & $54^{\circ} 08^{\prime} \mathrm{N} /$ & $54^{\circ} 09^{\prime} \mathrm{N} /$ & $54^{\circ} 12^{\prime} \mathrm{N} /$ & $54^{\circ} 03^{\prime} \mathrm{N} /$ & $54^{\circ} 05^{\prime} \mathrm{N} /$ & $54^{\circ} 09^{\prime} \mathrm{N} /$ & $54^{\circ} 13^{\prime} \mathrm{N} /$ \\
\hline & $07^{\circ} 55^{\prime} \mathrm{E}$ & $07^{\circ} 56^{\prime} \mathrm{E}$ & $07^{\circ} 58^{\prime} \mathrm{E}$ & $08^{\circ} 01^{\prime} \mathrm{E}$ & $07^{\circ} 56^{\prime} \mathrm{E}$ & $08^{\circ} 02^{\prime} \mathrm{E}$ & $08^{\circ} 03^{\prime} \mathrm{E}$ & $07^{\circ} 46^{\prime} \mathrm{E}$ \\
\hline Depth $(\mathrm{m})$ & 47.5 & 42.9 & 39.5 & 10.5 & 33.0 & 23.0 & 23.0 & 33.8 \\
\hline Sediment structure & \multicolumn{2}{|c|}{$\begin{array}{c}\text { medium sand, } \\
\text { shells, stones }\end{array}$} & \multicolumn{2}{|c|}{$\begin{array}{c}\text { medium sand, } \\
\text { partly gravel }\end{array}$} & \multicolumn{3}{|c|}{ mud and clay blended with fine sand } & $\begin{array}{l}\text { black clayed } \\
\text { mud }\end{array}$ \\
\hline $\begin{array}{l}\text { Content of organic } \\
\text { matter }(\%)\end{array}$ & 2.0 & 2.1 & 1.3 & 0.1 & 5.5 & 5.3 & 3.2 & 2.6 \\
\hline \multicolumn{9}{|l|}{ Ophiuroid species: } \\
\hline Acrocnida brachiata & - & - & - & - & 1 (low) & 1 (low) & - & $($ low $)$ \\
\hline Amphiura filiformis & - & 5 & 2 & - & 3 (medium) & - & 7 & 89 (low) \\
\hline Amphipholis squamata & 1 & - & - & - & - & - & - & 1 \\
\hline Ophiura albida & 11 & 22 (high) & 10 & 2 & 35 (high) & 86 (high) & 45 & (low) \\
\hline Ophiura ophiura & - & - & - & - & - & 6 (high) & - & - \\
\hline Ophiothrix fragilis & (low) & - & - & - & - & - & - & - \\
\hline
\end{tabular}

was maintained in separate flow-through containers for further processing. Sorting was carried out in the laboratories of the Marine Station 'Biologische Anstalt Helgoland' (BAH). Ophiuroids were identified to species level and their respective abundances per station recorded. For photographic documentation, the individuals were anaesthetized in isotonic magnesium sulphate solution, fixed in $70 \%$ ethanol and dried.

The sediment samples retained from each station were dried and sieved to fractions covering a series of seven mesh sizes between 3600 and $125 \mu \mathrm{m}$. Subsequently, the sediment types were defined according to the Wentworth grade classification (Holme \& McIntyre, 1984). According to these authors, fractions exceeding $2000 \mu \mathrm{m}$ relate to cobbles, pebbles and granules, whereas fractions between 2000 and $125 \mu \mathrm{m}$ refer to different grades of sands. Fractions below $125 \mu \mathrm{m}$ apply to very fine sands, silt and clay, respectively. The dominating fraction (referring to at least $25 \%$ in each sample) was used for identification of sediment type. Ash free dry weight of sediment was used to calculate the content of organic matter in each sample.

Literature dating back to 1875 was reviewed for qualitative and quantitative data on ophiuroids recorded for the inner German Bight (an area of approximately $40,000 \mathrm{~km}^{2}$, limited by the German North Sea coast, latitude $55^{\circ} 05^{\prime} \mathrm{N}$ and longitude $6^{\circ} 40^{\prime} \mathrm{E}$; Figure 1). The comparison of literature data is problematic because the data were not collected for this special purpose. In the historical literature, references of abundance are often given verbally, and thus imply a high amount of subjective assessment of the actual situation. Furthermore, the investigations widely differed in temporal and spatial coverage as well as in sorting method. Due to limitation on comparative analyses of the species' quantities over time, the individual references of abundance are discussed separately.

\section{RESULTS}

Sediment characteristics

At Stations 1-4, analysis of sediment revealed mainly fine and medium sands with dominating grain sizes between 500 and $250 \mu \mathrm{m}$, occasionally blended with coarser sediment fractions (Figure 1; Table 1). A particular composition of medium sands, shells and stones was determined for the 'Helgoländer Tiefe Rinne' (Stations 1 and 2), a depression south of Helgoland reaching a unique depth in the German Bight of about $60 \mathrm{~m}$. Conversely, Stations 5-8 were basically composed of muddy and clayed sediments with grain sizes $<125 \mu \mathrm{m}$, partly blended with fine sand. Basically, the stations can be divided in showing either coarse sediment types (Stations 1-4) or fine substrata (Stations 5-8), respectively. The sediment characteristics defined in this study are in accordance with those given by Stripp (1969) and Salzwedel et al. (1985). Content of organic matter was three times higher in muddy substrata from Stations $5-8(4.2 \pm 1.5 \%)$ than in sandy sediments from Stations $1-4(1.4 \pm 0.9 \%)$ (Table 1$)$.

\section{Species composition}

In total, six ophiuroid species belonging to three families (Amphiuridae, Ophiothrichidae and Ophiuridae) were identified off Helgoland (Table 1). Two burrowing species, Acrocnida brachiata (Montagu, 1804) (Figure 2A) and Amphiura filiformis (Müller, 1776) (Figure 2B), as well as three epibenthic brittle stars, Amphipholis squamata (Delle Chiaje, 1829) (Figure 2C), Ophiura albida Forbes, 1839 (Figure 2E) and Ophiura ophiura (Linnaeus, 1758) (Figure $2 \mathrm{~F}$ ) were found in various grab samples at different stations. Apart from A. squamata, all species were sampled by dredging as well. Ophiothrix fragilis (Abildgaard, 1789) (Figure 2D), however, was exclusively found in dredge samples at the Tiefe Rinne (Station 1).

The most common species, Ophiura albida, occurred on all types of sediment. Highest abundances of this species, however, reaching a maximum of 286 ind $\mathrm{m}^{-2}$ (Station 6 ), were recorded on fine (muddy) substrate (Stations 5, 6 and 7) rather than on coarse (sandy) sediments (Table 1). Dredge samples generally revealed high densities of O. albida. Amphiura filiformis was highly abundant on muddy substrata as well. While coarser sediments revealed low numbers of individuals, a maximum of about 300 ind $\mathrm{m}^{-2}$ was found on a muddy location north-west 


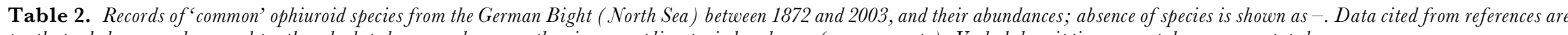
partly pooled mean values, and partly calculated mean values over the given sampling period and area (see comments). Verbal descriptions were taken over as stated.

\begin{tabular}{|c|c|c|c|c|c|c|c|c|}
\hline \multicolumn{9}{|c|}{ 'Common species' } \\
\hline Acrocnida brachiata & Amphiura filiformis & Amphipholis squamata & Ophiothrix fragilis & Ophiura albida & Ophiura ophiura & Comment & Year of study & Reference \\
\hline- & $\begin{array}{l}\text { Medium (found at } \\
1 \text { station in the } \\
\text { given range) }\end{array}$ & - & $\begin{array}{l}\text { Rare (found at } \\
1 \text { station in the } \\
\text { given range) }\end{array}$ & $\begin{array}{l}\text { Medium to } \\
\text { frequent (found } \\
\text { at } 5 \text { stations in } \\
\text { the given range) }\end{array}$ & $\begin{array}{l}\text { Rare to frequent } \\
\text { (found at } 4 \\
\text { stations in the } \\
\text { given range) }\end{array}$ & $\begin{array}{c}\text { No legend to } \\
\text { categories } \\
\text { available }\end{array}$ & 1872 & $\begin{array}{l}\text { Möbius \& Bütschli, } \\
\quad 1875\end{array}$ \\
\hline- & $\begin{array}{l}\text { Rare (found at } 3 \\
\text { stations in the } \\
\text { given range) }\end{array}$ & $\begin{array}{l}\text { Rare (found at } \\
1 \text { station in the } \\
\text { given range) }\end{array}$ & $\begin{array}{l}\text { Rare (found at } \\
1 \text { station in the } \\
\text { given range) }\end{array}$ & $\begin{array}{l}\text { Very frequent } \\
\text { (found at } 13 \\
\text { stations in the } \\
\text { given range) }\end{array}$ & $\begin{array}{l}\text { Rare to medium } \\
\text { (found at } 6 \\
\text { stations in the } \\
\text { given range) }\end{array}$ & $\begin{array}{l}\text { Categories of } \\
\text { abundance: } \\
\text { rare }=1-2 \text { ind, } \\
\text { medium }=3-6 \text { ind, } \\
\text { frequent }=\text { many } \\
\text { ind, very } \\
\text { frequent }=\text { very } \\
\text { many ind }\end{array}$ & 1889-1890 & $\begin{array}{l}\text { Meissner \& Collin, } \\
1894\end{array}$ \\
\hline $\begin{array}{l}\text { Rare (found at } 1 \\
\text { position along } \\
\text { the borders of } \\
\text { the given range) }\end{array}$ & $\begin{array}{l}\text { Very frequent } \\
\text { (found at } 7 \\
\text { positions in the } \\
\text { given range) }\end{array}$ & - & $\begin{array}{l}\text { Very frequent, } \\
\text { but patchy } \\
\text { distributed in the } \\
\text { North Sea (found } \\
\text { at } 8 \text { positions in } \\
\text { the given range) }\end{array}$ & $\begin{array}{l}\text { Very frequent } \\
\text { (found at } 11 \\
\text { positions in the } \\
\text { given range) }\end{array}$ & $\begin{array}{l}\text { Frequent (found } \\
\text { at } 3 \text { positions in } \\
\text { the given range) }\end{array}$ & $\begin{array}{l}21 \text { of } 170 \text { sampled } \\
\text { positions are in } \\
\text { the given ranges of } \\
\text { the present work }\end{array}$ & $1902-1907$ & $\begin{array}{l}\text { Süßbach \& } \\
\quad \text { Breckner, } 1911\end{array}$ \\
\hline- & $\begin{array}{l}\text { Between } 0.4 \text { and } \\
583.0 \text { ind } \mathrm{m}^{-2} \text { in } \\
\text { grab samples from } \\
4 \text { of } 5 \text { different } \\
\text { faunal areas }\end{array}$ & 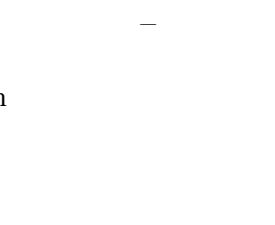 & - & $\begin{array}{l}\text { Between } 0.6 \text { and } \\
46.0 \text { ind } \mathrm{m}^{-2} \text { in } \\
\text { grab samples from } \\
3 \text { of } 5 \text { different } \\
\text { faunal areas }\end{array}$ & $\begin{array}{l}\text { Between } 1 \text { and } 17.8 \\
\text { ind } \mathrm{m}^{-2} \text { in grab } \\
\text { samples from } 3 \text { of } \\
5 \text { different faunal } \\
\text { areas }\end{array}$ & $\begin{array}{l}\text { Study of faunal } \\
\text { associations in the } \\
\text { German Bight. } \\
\text { Mean values per } \\
\mathrm{m}^{-2} \text { are given (no } \\
\text { SD available) }\end{array}$ & 1923-1924 & Hagmeier, 1925 \\
\hline- & $\begin{array}{l}\text { Between } 5.0 \\
\quad( \pm 10.0) \text { and } \\
632.2( \pm 233.7) \\
\text { ind } \mathrm{m}^{-2} \text { in grab } \\
\text { samples }\end{array}$ & $\begin{array}{l}15 \text { individuals } \\
\text { found in a single } \\
\text { van Veen grab } \\
\left(0.1 \mathrm{~m}^{2}\right)\end{array}$ & $\begin{array}{l}\text { Found in dredges } \\
\text { (no precise data); } \\
\text { further descrip- } \\
\text { tion: 'not as } \\
\text { frequent as the } \\
\text { other brittle stars } \\
\text { found in this } \\
\text { study' }\end{array}$ & $\begin{array}{l}\text { Between } 7.5( \pm 9.6) \\
\text { and } 136.7 \\
( \pm 92.9) \text { ind } \mathrm{m}^{-2} \\
\text { in grab samples as } \\
\text { well as in dredges } \\
\text { (no precise data) }\end{array}$ & - & $\begin{array}{l}\text { Study site: 'Tiefe } \\
\text { Rinne', } 35 \text { stations } \\
\text { were sampled in a } \\
\text { total of } 4 \text { different } \\
\text { faunal associa- } \\
\text { tions. Mean } \\
\text { values calculated } \\
\text { per faunal associa- } \\
\text { tion }\end{array}$ & 1938 & Caspers, 1938 \\
\hline
\end{tabular}


Table 2. (Continued).

\begin{tabular}{|c|c|c|c|c|c|c|c|c|}
\hline \multicolumn{9}{|c|}{ 'Common species' } \\
\hline Acrocnida brachiata & Amphiura filiformis & Amphipholis squamata & Ophiothrix fragilis & Ophiura albida & Ophiura ophiura & Comment & Year of study & Reference \\
\hline- & $\begin{array}{l}\text { Between } 34.4 \\
\quad( \pm 28.8) \text { and } \\
123.0( \pm 144.9) \\
\text { ind } \mathrm{m}^{-2} \text { in grab } \\
\text { samples }\end{array}$ & - & $\begin{array}{l}10 \text { individuals } \\
\text { found in dredges } \\
\text { (no precise data); } \\
\text { 'in great quanti- } \\
\text { ties between } \\
\text { oyster shell } \\
\text { fragments' }\end{array}$ & $\begin{array}{l}\text { Between } 29.0 \\
\quad( \pm 31.4) \text { and } 45.6 \\
\quad( \pm 63.1) \text { ind } \mathrm{m}^{-2} \\
\text { in grab samples, } \\
\text { found in dredges } \\
\text { as well (no precise } \\
\text { data) }\end{array}$ & 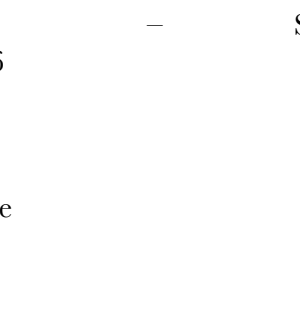 & $\begin{array}{l}\text { Study site: } \\
\text { 'Austernbank'. } 44 \\
\text { stations were } \\
\text { sampled. Mean } \\
\text { values are calcu- } \\
\text { lated from a small } \\
\text { number of stations } \\
\text { presented by } \\
\text { Caspers }\end{array}$ & 1938 & Caspers, 1950 \\
\hline- & $\begin{array}{l}0.01( \pm 0.03) \\
\text { ind } \mathrm{m}^{-2}\end{array}$ & $\begin{array}{l}0.01( \pm 0.03) \\
\quad \text { ind } \mathrm{m}^{-2}\end{array}$ & - & $\begin{array}{c}5.4( \pm 7.9) \text { ind } \\
\mathrm{m}^{-2}\end{array}$ & $\begin{array}{l}13.9( \pm 13.5) \\
\text { ind } \mathrm{m}^{-2}\end{array}$ & $\begin{array}{l}\text { Pooled mean } \\
\text { values for } 9 \\
\text { stations sampled } \\
\text { during the given } \\
\text { time period }\end{array}$ & $1950-1974$ & Ziegelmeier, 1987 \\
\hline- & $\begin{array}{l}\text { Between 'rare' and } \\
30.0( \pm 19.0) \text { ind } \\
\mathrm{m}^{-2} \text { on } 2 \text { of } 5 \\
\text { stations and in } \\
2 \text { of } 4 \text { faunal } \\
\text { associations }\end{array}$ & $\begin{array}{l}42.0( \pm 44.7) \text { ind } \\
\mathrm{m}^{-2} \text { on } 1 \text { of } 5 \\
\text { stations }\end{array}$ & $\begin{array}{l}\text { 'Rare' in } 1 \text { of } 4 \\
\text { faunal } \\
\text { associations }\end{array}$ & $\begin{array}{l}\text { Between 'rare' and } \\
232.5( \pm 333.5) \\
\text { ind } \mathrm{m}^{-2} \text { on } 4 \text { of } \\
5 \text { stations and in } \\
3 \text { of } 4 \text { faunal } \\
\text { associations }\end{array}$ & $\begin{array}{l}\text { Between 'rare' } \\
\text { and } 9.0( \pm 9.9) \\
\text { ind } \mathrm{m}^{-2} \text { on } 3 \text { of } \\
5 \text { stations and in } \\
3 \text { of } 4 \text { faunal } \\
\text { associations }\end{array}$ & $\begin{array}{l}\text { Pooled mean values } \\
\text { for } 4 \text { stations and } \\
\text { from } 4 \text { different } \\
\text { faunal associations } \\
\text { (with } 17 \text { to } 34 \\
\text { stations each) }\end{array}$ & 1965-1966 & Stripp, 1969 \\
\hline $\begin{array}{l}2.3 \text { ind } \mathrm{m}^{-2} \text { (no } \\
\mathrm{SD} \text { available) }\end{array}$ & $\begin{array}{l}46.0 \text { ind } \mathrm{m}^{-2} \text { (no } \\
\mathrm{SD} \text { available) }\end{array}$ & $\begin{array}{l}0.4 \text { ind } \mathrm{m}^{-2} \text { (no } \\
\mathrm{SD} \text { available) }\end{array}$ & $\begin{array}{l}<1 \text { ind } \mathrm{m}^{-2}(21 \\
\text { individuals } \\
\text { found on a rock })\end{array}$ & $\begin{array}{l}84.1 \text { ind } \mathrm{m}^{-2}(\mathrm{no} \\
\mathrm{SD} \text { available) }\end{array}$ & $\begin{array}{l}23.3 \text { ind } \mathrm{m}^{-2} \text { (no } \\
\mathrm{SD} \text { available) }\end{array}$ & $\begin{array}{l}\text { Pooled mean values } \\
\text { for the German } \\
\text { Bight (from } 4 \\
\text { different faunal } \\
\text { associations) given } \\
\text { by Gerdes }\end{array}$ & $1969-1976$ & Gerdes, 1977 \\
\hline $\begin{array}{l}10.9( \pm 26.9) \text { ind } \\
\mathrm{m}^{-2} \text { on } 1 \text { of } 5 \\
\text { faunal associations }\end{array}$ & $\begin{array}{l}\text { Between }<1 \text { and } \\
296.6( \pm 343.8) \\
\text { ind } \mathrm{m}^{-2} \text { on } 4 \text { of } 5 \\
\text { faunal associations }\end{array}$ & $\begin{array}{l}<1 \text { ind } \mathrm{m}^{-2} \text { on } 2 \\
\text { of } 5 \text { faunal } \\
\text { associations }\end{array}$ & $\begin{array}{l}<1 \text { ind } \mathrm{m}^{-2} \text { on } 2 \\
\text { of } 5 \text { faunal } \\
\text { associations }\end{array}$ & $\begin{array}{l}\text { Between } 3.7 \\
\quad( \pm 11.0) \text { and } \\
263.1( \pm 297.6) \\
\text { ind } \mathrm{m}^{-2} \text { on } 5 \text { of } 5 \\
\text { faunal associations }\end{array}$ & $\begin{array}{l}\text { Between }<1 \text { and } \\
150.9( \pm 153.4) \\
\text { ind } \mathrm{m}^{-2} \text { on } 4 \text { of } 5 \\
\text { faunal associations }\end{array}$ & $\begin{array}{l}\text { Mean values per } \\
\text { faunal associations } \\
\text { (between } 7 \text { and } \\
\text { s } 26 \text { stations per } \\
\text { association) }\end{array}$ & 1975 & $\begin{array}{l}\text { Salzwedel et al., } \\
1985\end{array}$ \\
\hline $\begin{array}{l}6.5( \pm 10.9) \\
\quad \text { ind } \mathrm{m}^{-2}\end{array}$ & $\begin{array}{l}8.24( \pm 11.1) \\
\quad \text { ind } \mathrm{m}^{-2}\end{array}$ & - & - & $\begin{array}{l}70.72( \pm 67.1) \\
\text { ind } \mathrm{m}^{-2}\end{array}$ & $\begin{array}{l}15.52( \pm 51.8) \\
\quad \text { ind } \mathrm{m}^{-2}\end{array}$ & $\begin{array}{l}\text { Study site: stations } \\
\text { on transects in the } \\
\text { North Sea. Mean } \\
\text { values for all } \\
\text { stations sampled } \\
\text { in the given range } \\
\text { and time period }\end{array}$ & 1984-1988 & Niermann, 1997 \\
\hline
\end{tabular}


Table 2. (Continued).

\begin{tabular}{|c|c|c|c|c|c|c|c|c|}
\hline \multicolumn{9}{|c|}{ 'Common species' } \\
\hline Acrocnida brachiata & Amphiura filiformis & Amphipholis squamata & Ophiothrix fragilis & Ophiura albida & Ophiura ophiura & Comment & Year of study & Reference \\
\hline $\begin{array}{l}0.4( \pm 2.0) \\
\quad \text { ind } \mathrm{m}^{-2}\end{array}$ & $\begin{array}{l}6.4( \pm 20.6) \\
\quad \text { ind } \mathrm{m}^{-2}\end{array}$ & $\begin{array}{l}193.6( \pm 376.1) \\
\text { ind } \mathrm{m}^{-2}\end{array}$ & - & $\begin{array}{l}44.8( \pm 79.4) \\
\text { ind } \mathrm{m}^{-2}\end{array}$ & - & $\begin{array}{l}\text { Study site: 'Tiefe } \\
\text { Rinne'; mean } \\
\text { values per } 25 \\
\text { stations sampled }\end{array}$ & 1988-1989 & Berberich, 1989 \\
\hline $\begin{array}{l}\text { Low numbers at } 3 \\
\text { of } 6 \text { stations }\end{array}$ & $\begin{array}{l}\text { Low numbers at } \\
3 \text { of } 6 \text { stations }\end{array}$ & Present & $\begin{array}{l}\text { Found at } 2 \text { of } 6 \\
\text { stations }\end{array}$ & $\begin{array}{l}\text { Characterizing } \\
\text { species in dredges } \\
\text { at } 6 \text { stations } \\
\text { (dominance: } \\
1.28 \% \text { and } \\
7.40 \% \text { ) }\end{array}$ & $\begin{array}{l}\text { Characterizing } \\
\text { species in dredges } \\
\text { at } 4 \text { stations } \\
\text { (Dominance: } \\
2.07 \% \text { ) }\end{array}$ & $\begin{array}{l}\text { Study site: 'Stein- } \\
\text { grund'; } 6 \text { stations } \\
\text { sampled (absolute } \\
\text { data not } \\
\text { available) }\end{array}$ & 1991 & $\begin{array}{l}\text { Kühne \& Rachor, } \\
1996\end{array}$ \\
\hline $\begin{array}{l}<1 \text { ind } \mathrm{m}^{-2} \text { at } 3 \\
\text { of } 4 \text { stations }\end{array}$ & $\begin{array}{l}\text { Between }<1 \text { and } \\
1234( \pm 1072) \text { ind } \\
\mathrm{m}^{-2} \text { at } 4 \text { stations }\end{array}$ & - & - & $\begin{array}{l}\text { Between } 5( \pm 15) \\
\text { and } 176( \pm 99) \\
\text { ind } \mathrm{m}^{-2} \text { at } \\
4 \text { stations }\end{array}$ & $\begin{array}{l}\text { Between }<1 \text { and } \\
97( \pm 124) \text { ind } \\
\mathrm{m}^{-2} \text { at } 4 \text { stations }\end{array}$ & $\begin{array}{l}\text { Pooled mean values } \\
\text { from } 4 \text { stations } \\
\text { for the given time } \\
\text { period, given by } \\
\text { Schroeder }\end{array}$ & 1969-2000 & Schroeder, 2005 \\
\hline $\begin{array}{l}0.8( \pm 1.5) \text { ind } \\
\mathrm{m}^{-2} \text { in grab } \\
\text { samples and low } \\
\text { numbers in } \\
\text { dredges }\end{array}$ & $\begin{array}{l}44.2( \pm 102.4) \\
\text { ind } \mathrm{m}^{-2} \text { in grab } \\
\text { samples and low } \\
\text { to medium } \\
\text { numbers in } \\
\text { dredges }\end{array}$ & $\begin{array}{l}0.83( \pm 1.5) \text { ind } \\
\mathrm{m}^{-2} \text { in grab } \\
\text { samples }\end{array}$ & $\begin{array}{l}\text { Low numbers in } \\
\text { dredges }\end{array}$ & $\begin{array}{l}87.9( \pm 95.9) \text { ind } \\
\mathrm{m}^{-2} \text { in grab } \\
\text { samples and } \\
\text { generally high } \\
\text { numbers } \\
\text { especially in } \\
\text { dredges }\end{array}$ & $\begin{array}{l}2.5( \pm 7.1) \text { ind } \\
\mathrm{m}^{-2} \text { in grab } \\
\text { samples and } \\
\text { generally high } \\
\text { numbers in } \\
\text { dredges }\end{array}$ & $\begin{array}{l}\text { Mean values from } \\
8 \text { stations sampled }\end{array}$ & 2003 & Present study \\
\hline
\end{tabular}

SD, standard deviation. 


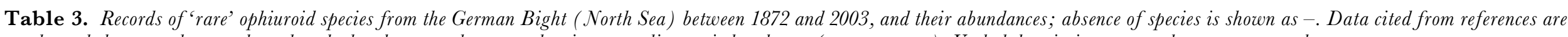
partly pooled mean values, and partly calculated mean values over the given sampling period and area (see comments). Verbal descriptions were taken over as stated.

\begin{tabular}{|c|c|c|c|c|c|c|}
\hline \multicolumn{7}{|c|}{ 'Rare species' } \\
\hline Amphiura chiajei & Ophiopholis aculeata & Ophiocten affinis & Ophiura sarsi & Comment & Year of study & Reference \\
\hline- & $\begin{array}{l}\text { Medium (found at } \\
1 \text { station in the } \\
\text { given range) }\end{array}$ & - & $\begin{array}{l}\text { Medium (found at } \\
1 \text { station in the } \\
\text { given range) }\end{array}$ & $\begin{array}{l}\text { No legend to categories } \\
\text { available }\end{array}$ & 1872 & Möbius \& Bütschli, 1875 \\
\hline- & - & $\begin{array}{l}\text { Very frequent (found at } \\
1 \text { station in the given } \\
\text { range) }\end{array}$ & - & $\begin{array}{l}\text { Categories of abundance: } \\
\text { rare }=1-2 \text { ind, } \\
\text { medium }=3-6 \text { ind, } \\
\text { frequent }=\text { many ind, } \\
\text { very frequent = very } \\
\text { many ind }\end{array}$ & 1889-1890 & Meissner \& Collin, 1894 \\
\hline $\begin{array}{l}\text { Rare (found at } 3 \text { positions } \\
\text { in the given range) }\end{array}$ & - & $\begin{array}{l}\text { Rare (found at } 2 \text { positions } \\
\text { in the given range) }\end{array}$ & - & $\begin{array}{l}21 \text { of } 170 \text { sampled posi- } \\
\text { tions are in the given } \\
\text { ranges of the present } \\
\text { work }\end{array}$ & $1902-1907$ & Süßbach \& Breckner, 1911 \\
\hline $\begin{array}{l}0.6 \text { ind } \mathrm{m}^{-2} \text { in grab } \\
\text { samples from } 1 \text { of } 5 \\
\text { different faunal areas } \\
\text { (sandy substrata) (no } \\
\text { SD available) }\end{array}$ & - & - & - & $\begin{array}{l}\text { Study of faunal associa- } \\
\text { tions in the German } \\
\text { Bight. Mean values per } \\
\mathrm{m}^{-2} \text { are given (no SD } \\
\text { available) }\end{array}$ & 1923-1924 & Hagmeier, 1925 \\
\hline- & - & - & - & & 1938 & Caspers, 1938 \\
\hline- & - & - & - & & 1938 & Caspers, 1950 \\
\hline- & - & - & - & & 1950-1974 & Ziegelmeier, 1987 \\
\hline- & - & - & - & & $1965-1966$ & Stripp, 1969 \\
\hline- & - & - & - & & 1969-1976 & Gerdes, 1977 \\
\hline- & - & - & - & & 1975 & Salzwedel et al., 1985 \\
\hline- & - & - & - & & 1984-1988 & Niermann, 1997 \\
\hline- & - & - & - & & 1988-1989 & Berberich, 1989 \\
\hline- & - & - & - & & 1991 & Kühne \& Rachor, 1996 \\
\hline $\begin{array}{l}<1 \text { ind } \mathrm{m}^{-2} \text { at } 1 \text { of } \\
\quad 4 \text { stations }\end{array}$ & - & - & - & $\begin{array}{l}\text { Pooled mean values from } \\
4 \text { stations for the given } \\
\text { time period, given by } \\
\text { Schroeder }\end{array}$ & 1969-2000 & Schroeder, 2005 \\
\hline- & - & - & - & & 2003 & Present study \\
\hline
\end{tabular}

SD, standard deviation. 
of Helgoland (Station 8). Dredges revealed low to medium densities. Low numbers in grab samples as well as in dredges and only at a few sampling sites were recorded for Acrocnida brachiata, Amphipholis squamata, Ophiothrix ophiura and O. fragilis (Table 1).

\section{DISGUSSION}

Our qualitative results are in agreement with those of previous investigations in the inner German Bight over the past decades (Möbius \& Bütschli, 1875; Meissner \& Collin, 1894; Süßbach \& Breckner, 1911; Hagmeier, 1925; Caspers, 1938, 1950; Stripp, 1969; Gerdes, 1977; Salzwedel et al., 1985; Ziegelmeier, 1987; Berberich, 1989; Kühne \& Rachor, 1996; Niermann, 1997; Schroeder, 2005; Tables 2 \& 3).

Süßbach \& Breckner (1911) recorded Acrocnida brachiata from the outer limits of the inner German Bight in 1904. After a long gap in time and the literature, it was recorded again in 1975 by Gerdes (1977). Since then it has been found regularly, although in fairly low to moderate abundances on muddy or very fine grained substrata (Salzwedel et al., 1985; Berberich, 1989; Kühne \& Rachor, 1996; Niermann, 1997; Schroeder, 2005; this study). Ursin (1960) refers to temperature as an important factor limiting this species' distribution. The author describes its absence from regions with summer temperatures below $10^{\circ} \mathrm{C}$ and winter temperatures below $3^{\circ} \mathrm{C}$. In the inner German Bight the lowest monthly mean temperature (usually in February) basically has not dropped below $2.7^{\circ} \mathrm{C}$ in the past 20 years (Franke et al., 1999) and has been significantly higher than in previous decades (Wiltshire \& Manly, 2004). Thus, increased winter temperatures might have promoted a range expansion of A. brachiata. In their studies, Webb \& Tyler (1985), Bourgoin \& Guillou (1990) and Bourgoin et al. (1991) identified delimited annual spawning processes as well as shortened larval stages and abbreviated or even missing pelagic phases in the life cycle of $A$. brachiata. This might help in explaining the slow but steady expansion and colonization toward the inner German Bight observed over the past 30 years.

Abundances of Amphiura filiformis are apparently very inconsistent over time (Table 2). This species was recorded in highest abundances with mean values between 297 ind $\mathrm{m}^{-2}$ (Salzwedel et al., 1985) and increased densities of 590 ind $\mathrm{m}^{-2}$ (Thatje \& Gerdes, 1997) on muddy substrata of the Amphiura filiformis association sensu Salzwedel et al. (1985). Stripp (1969), however, revealed only 5.1 ind $\mathrm{m}^{-2}$ in his equivalent Echinocardium cordatum (Pennant, 1777)-Amphiura filiformis association. Similar values for $A$. filiformis were reported in the same study from all other faunal communities on different types of sediment as well. In addition, Berberich (1989) found only 16 specimens on five of 25 stations sampled in the Tiefe Rinne. Variations in abundance of $A$. filiformis certainly refer to the different locations under study. The species' infaunal lifestyle in fine grain-sized sediments may explain the low densities or absence on coarser sediments or hard bottom substrata. Apart from sedimental aspects, however, variability in abundance over time may also be due to sensitivity to cold winter temperatures (Ursin, 1960). Even though this species may tolerate winter temperatures down to $3-4^{\circ} \mathrm{C}$, entire populations might perish if temperatures drop below this limit. Extinctions due to extreme winters, though, are apparently followed by a rapid recovery of populations (Ursin, 1960). This is ensured by larval transport from abundant populations of this species in the warmer south-west part of the North Sea. Thatje \& Gerdes (1997), for example, regarded an observed patchy distribution and high numbers of juvenile A. filiformis during their study as a direct consequence of an ongoing recruitment process.

Ophiura albida was recorded continuously on various types of sediment by all authors listed in Table 2 . Though abundances might differ between 'rare' and very high densities (Table 2; Stripp, 1969), this species appears to be highly abundant and very common in the German Bight. Although presumably not as abundant as O. albida, O. ophiura is found regularly in moderate to high densities on various substrata. In the Tiefe Rinne and at the former 'Helgoländer Austernbank', however, this species was lacking (Caspers, 1938, 1950; Berberich, 1989). Low abundances of $O$. ophiura in the present study refer to the relatively small area covered by grab samples (Table 2). The actual situation is probably reflected better by the dredge samples revealing high abundances (Table 2). Opposed to their amphiurid relatives, both epibenthic ophiurid species appear to be quite robust and rather mobile brittle stars. They exhibit a wide range of different feeding mechanisms, e.g. deposit feeding, taking advantage of the high amount of associated organic matter, scavenging or predation (Feder, 1981; Warner, 1982). Further, Sköld (1998) identified a variety of different defence mechanisms against potential predators, e.g. burrowing and rapid escape. These features make them rather opportunistic towards different conditions, and thus may explain for their success in comparably high densities over time.

Amphipholis squamata was reported on different sediments less regularly and in very low numbers (Meissner \& Collin, 1894; Caspers, 1938; Stripp, 1969; Gerdes, 1977; Salzwedel et al., 1985; Ziegelmeier, 1987; present study). Berberich (1989), however, recorded a mean value of nearly 200 ind $\mathrm{m}^{-2}$ from 25 stations in the Tiefe Rinne, which is characterized by a mixture of various types of substrata. Amphipholis squamata is a hermaphrodite and brooding species. It releases its offspring at a crawl-away juvenile stage and therefore does not disperse through planktonic larvae. Accordingly, slow and irregular recolonization processes may take place, for example after extreme mortality events. Various authors suggest that dispersal occurs through passive transport on other animals, e.g. medusae or by rafting on macroalgae (Highsmith, 1985; Edgar, 1987; O’Hara, 1998; Thiel \& Gutow, 2005). The species' mode of reproduction as well as its passive dispersal may explain the irregular findings and generally low abundances in the inner German Bight, although $A$. squamata is distributed world-wide. Furthermore, its preference for cryptic habitats such as crevices in rockpools or holdfasts of macroalgae may affect its occurrence on soft bottom sediments.

The occurrence of Ophiothrix fragilis in the inner German Bight is comparatively variable as well. Few studies revealed high numbers. Generally though, abundances are rather low or refer to single specimens often 
found on hard bottoms. In addition, it seems as if abundances were somewhat higher prior to 1950. This however, is difficult to interpret, since references of abundance are given in verbal expression for the respective period of time and thus do not allow for an objective comparison. Ophiothrix fragilis is a rheophilic suspension feeder and therefore dependent on high-current habitats where it can form dense aggregations exceeding 2000 ind $\mathrm{m}^{-2}$ (Warner, 1971). These aggregations act as local barriers to strong currents creating fronts of suspended matter, which allow for enhanced feeding success and eased fertilization (Hughes, 1998). Ophiothrix fragilis can be found in (micro-) habitats such as rocks, stones, sponges or corals (e.g. Alcyonium digitatum Linnaeus, 1758). These habitats allow for an elevated positioning and the avoidance of decelerated bottom-near currents. Where flow velocity is high enough, this species can be found on soft bottom sediments as well. These conditions, however, seem to be rather rare on the prevailing soft bottom sediments in the inner German Bight. Hence, specimens of O. fragilis presumably occur only sporadically in the inner German Bight as isolated individuals or in small patchy distributed populations.

Five out of ten species recorded in previous investigations in the inner German Bight over the past 130 years, were confirmed in this study. From this point of view, Acrocnida brachiata must be treated as a newcomer since the late 1970 s, possibly as an effect of climate change. Yet, due to its continuous occurrence since then, all ophiuroid species recorded in the present study can be referred to as 'common' and regularly found species in the inner German Bight. Our quantitative results correspond roughly to the general patterns of abundance depicted by previous investigators. Increasing or decreasing trends in abundance over time, however, cannot be inferred from the reviewed data due to the way they were presented. Non-systematic and sporadic investigations over longer periods of time do not allow for an unequivocal interpretation of changes in abundance (Franke \& Gutow, 2004). However, different anthropogenic or biological factors may explain irregularities in records as well as in abundance over the reviewed period of time.

Information on further brittle star species in the inner German Bight refer to Amphiura chiajei Forbes 1843, Ophiopholis aculeata (Linnaeus 1767), Ophiocten affinis (Lütken 1858) and Ophiura sarsi Lütken 1858, and was provided mainly for the period 1872 to 1924 by Möbius \& Bütschli (1875), Meissner \& Collin (1894), Süßbach \& Breckner (1911) and Hagmeier (1925). Again, verbal expression in reference to abundance makes their densities uncertain to assess, but seemingly refers to scarce or moderate occurrence. This is particularly true for two singular records of $A$. chiajei in 1977 and 1983 with only two individuals each found in the entire sampling procedures (Schroeder, 2005). Amphiura chiajei has ecological demands very similar to $A$. filiformis. While the latter species preferably feeds as a suspension feeder and relies on horizontal transport of suspended matter, A. chiajei is considered a deposit feeder (Duineveld et al., 1987; Hollertz et al., 1998). Therefore, A. chiajei depends on habitats, which allow for a deposition of fine particulate matter (Buchanan, 1964). The surface sediments in the inner German Bight are fairly mobile (Becker et al.,
1992). Due to irregular currents, wave action, tidal effects and heavy beam trawl fishery, substrata are resuspended to a large extent and carried away. Suboptimal physical conditions might partly explain the species' absence from the inner German Bight.

Since almost all records of A. chiajei, Ophiopholis aculeata, Ophiocten affinis and Ophiura sarsi date back to the period prior to 1924, these species most probably can be considered as incidental visitors in the inner German Bightperhaps due to unusually favourable conditions at that time. Süßbach \& Breckner (1911) provided very detailed descriptions of the species they found. Nonetheless, in the historical literature misidentifications and misleading synonyms must be taken into consideration. However, according to the species' overall short-term occurrence as well as their apparently low abundances, they are referred to as 'rare' species in the inner German Bight.

Apart from Amphipholis squamata which has a cosmopolitan distribution along the littoral zones of warm and temperate as well as arctic and antarctic waters (Unno, 2000), all ophiuroid species recorded for the inner German Bight are common representatives of the boreo-lusitanian fauna (Hyman, 1955) showing wide distributional ranges.

Due to a strong continental influence, coastal waters, e.g. those of the inner German Bight, show a higher annual variability in temperature and salinity compared to the central North Sea (Ursin, 1960). Suitable winter temperatures may occur in the inner German Bight for all of the species mentioned in Tables 2 and 3. Conversely, unfavourably high summer temperatures might play an important role in reproduction and thus in distribution of some species with northerly focused ranges, e.g. Ophiopholis aculeata. This might explain the broad absence for some of the 'rare' titled species from the inner German Bight. Most of the 'common' representatives, such as Ophiura albida, O. ophiura and Amphiura filiformis are highly abundant species in the German Bight. They have been described over time as characterizing and even dominating different faunal associations. Their high abundances and share in biomass as well as their broad ecological ranges indicate very low extinction probabilities and thus account for a more or less steady ophiuroid species composition over time.

Rees et al. (1999) showed a decrease of benthic biodiversity in the southern North Sea following the broad coastlines from the English Channel into the southeastern areas of the North Sea. While sediment analyses revealed higher amounts of coarser grounds along the Channel, the south-eastern parts of the North Sea are characterized by mainly sandy and muddy areas. The authors suggested substratum type to be the 'main structuring force' in distribution. From this point of view, the inner German Bight may be an unsuitable area for potential arrivals of many species from the Channel area. Different authors, however, pointed out, that various investigations on benthic communities in the inner German Bight revealed serious shifts in species composition over time due to anthropogenic or natural changes in the environment (Thatje \& Gerdes, 1997; Franke \& Gutow, 2004; Reichert \& Buchholz, 2006). In addition to changes in the species spectrum, a general trend towards increased biomass and density was shown in a review of 
long-term changes in the North Sea benthos by Kröncke (1995) and by Thatje \& Gerdes (1997). The authors particularly refer to small short-lived species such as many polychaetes, bivalves, ophiuroids-especially A. filiformis - and echinoids. Anthropogenic effects such as eutrophication were considered most important. While changes in the ophiuroids abundances can be observed, it is yet difficult to distinguish between effects of anthropogenic and natural factors, and between mere fluctuations and serious long-term trends in ecosystem structure and function.

Qualitative changes in the species composition of ophiuroids over time refer to the arrival of Acrocnida brachiata in the 1970s. Some occasional findings of rare titled species have been reported during the time period investigated. These findings, however, cannot account for changes in ophiuroid species diversity over time. Thus, a fairly stable composition of brittle stars can be stated for the inner German Bight over the past 130 years.

The present study is part of a diploma thesis carried out at the Biologische Anstalt Helgoland. Our thanks go to the Foundation Alfred Wegener Institute for Polar and Marine Research and in particular to the staff of the Marine Station on the Island of Helgoland for providing research facilities and personal support - especially the crew of the MS 'Uthörn'. Special thanks to Lars Gutow for fruitful comments on the manuscript.

\section{REFERENCES}

Becker, G.A., Dick, S. \& Dippner, J.W., 1992. Hydrography of the German Bight. Marine Ecology Progress Series, 91, 9-18.

Benoit, J., 1998. Langzeiteffekte solarer UV-Strahlung auf marine Braunalgen der Gattung Fucus, insbesondere deren frühe ontogenetische Stadien, und die mögliche Bedeutung für die Zonierung im Eulitoral von Helgoland. Diploma thesis, Universität Freiburg, Germany, unpublished.

Berberich, D., 1989. Vergleichende Untersuchungen zur Artenzusammensetzung, Abundanz und Biomasse des Makrozoobenthos der Helgoländer Tiefen Rinne von 1936/37 und 1988/89. Diploma thesis, Technische Hochschule Darmstadt, Germany, unpublished.

Bourgoin, A. \& Guillou, M., 1990. Variations in the reproductive cycle of Acrocnida brachiata (Echinodermata: Ophiuroidea) according to environment in the Bay of Douarnenez (Brittany). Fournal of the Marine Biological Association of the United Kingdom, 70, 57-66.

Bourgoin, A., Guillou, M. \& Glémarec, M., 1991. Environmental instability and demographic variability in Acrocnida brachiata (Echinodermata: Ophiuroidea) in Douarnenez Bay (Brittany: France). Marine Ecology, 12, 89-104.

Buchanan, J.B., 1964. A comparative study of some features of the biology of Amphiura filiformis and Amphiura chiajei (Ophiuroidea) considered in relation to their distribution. fournal of the Marine Biological Association of the United Kingdom, 44, 565-576.

Caspers, H., 1938. Die Bodenfauna der Helgoländer Tiefen Rinne. Helgoländer Wissenschaftliche Meeresuntersuchungen, 2, $1-112$.

Caspers, H., 1950. Die Lebensgemeinschaft der Helgoländer Austernbank. Helgoländer Wissenschaftliche Meeresuntersuchungen, 3, 120-169.

Duineveld, G.C.A., Künitzer, A. \& Heyman, R.P., 1987. Amphiura filiformis (Ophiuroidea: Echinodermata) in the North Sea. Distribution, present and former abundance and size composition. Netherlands Fournal of Sea Research, 21, 317-329.
Edgar, G.J., 1987. Dispersal of faunal and floral propaguels associated with drifting Macrocystis pyrifera plants. Marine Biology, 95, 599-610.

Feder, H.M., 1981. Aspects of the feeding biology of the brittle star Ophiura texturata. Ophelia, 20, 215-235.

Franke, H.D. \& Gutow, L., 2004. Long term changes in the macrozoobenthos around the rocky island of Helgoland (German Bight, North Sea). Helgoland Marine Research, 58, 303-310.

Franke, H.D., Gutow, L. \& Janke, M., 1999. The recent arrival of the oceanic isopod Idotea metallica Bosc off Helgoland (German Bight, North Sea): an indication of a warming trend in the North Sea? Helgoländer Meeresuntersuchungen, 52, 347-357.

Gerdes, D., 1977. Zur Verteilung der Echinodermen in der Deutschen Bucht. Diploma thesis, Institut für Meereskunde, Kiel, Germany, unpublished.

Hagmeier, A., 1925. Vorläufiger Bericht über die vorbereitenden Untersuchungen der Bodenfauna der Deutschen Bucht mit dem Petersen-Bodengreifer. Berichte der Deutschen Wissenschaftlichen Kommission für Meeresforschung, N.F. 1, 247272.

Highsmith, R.C., 1985. Floating and algal rafting as potential dispersal mechnanism in brooding invertebrates. Marine Ecology Progress Series, 25, 169-179.

Hollertz, K., Sköld, M. \& Rosenberg, R., 1998. Interactions between two deposit feeding echinoderms: the spatangoid Brissopsis lyrifera (Forbes) and the ophiuroid Amphiura chiajei Forbes. Hydrobiologia, 375/376, 287-295.

Holme, N.A. \& McIntyre, A.D., 1984. Methods for the study of marine benthos, 2nd edn. Oxford: Blackwell Scientific Publications.

Hughes, D.J., 1998. Subtidal brittlestar beds, vol. IV. An overview of dynamics and sensitivity characteristics for conservation management of marine SACs. Scottish Association for Marine Science (UK Marine SACs Project), 78 pp.

Hyman, L., 1955. The invertebrates-Echinodermata, vol. IV. New York: McGraw-Hill Book Company Inc.

Kröncke, I., 1995. Long-term changes in North Sea Benthos. Senckenbergiana Maritima, 26, 73-80.

Kühne, S. \& Rachor, E., 1996. The macrofauna of a stony sand area in the German Bight (North Sea). Helgoländer Meeresuntersuchungen, 50, 433-452.

Meissner, M. \& Collin, A., 1894. Beiträge zur Fauna der südöstlichen und östlichen Nordsee, Teil II. Echinodermen. Helgoländer Wissenschaftliche Meeresuntersuchungen, 1, 329-346.

Möbius, K. \& Bütschli, O., 1875. Zoologische Ergebnisse der Nordseefahrt. Teil IV. Echinodermata. Fahresbericht der Kommission zur Wissenschaftichen Untersuchung der Deutschen Meere in Kiel für die Jahre 1872-73, 2-3, 143-152.

Niermann, U., 1997. Macrobenthos of the south-eastern North Sea during 1984-88. Berichte der Biologischen Anstalt Helgoland, 13, $1-144$.

O'Hara, T., 1998. Origin of Macquarie Island echinoderms. Polar Biology, 20, 143-151.

Rees, H.L., Pendle, M.A., Waldock, R., Limpenny, D.S. \& Boyd, E.S., 1999. A comparison of benthic biodiversity in the North Sea, English Channel, and Celtic Seas. ICES Journal of Marine Science, 56, 228-246.

Reichert, K. \& Buchholz, F., 2006. Changes in the macrozoobenthos of the intertidal zone at Helgoland (German Bight, North Sea): a survey of 1984 repeated in 2002. Helgoland Marine Research, in press.

Reise, K., Gollasch, S. \& Wolff, W.J., 1999. Introduced marine species of the North Sea coasts. Helgoländer Meeresuntersuchungen, 52, 219-234.

Salzwedel, H., Rachor, E. \& Gerdes, D., 1985. Benthic macrofauna communities in the German Bight. Veröffentlichungen des Instituts für Meeresforschung in Bremerhaven, 20, 199-267. 
Schroeder, A., 2005. Community dynamics and development of softbottom macrozoobenthos in the German Bight/North Sea) 1969-2000. Berichte zur Polar- und Meeresforschung, 494, 1181.

Sköld, M., 1998. Escape responses in four epibenthic brittle stars (Ophiuroidea: Echinodermata). Ophelia, 49, 163-179.

Stripp, K., 1969. Die Assoziationen des Benthos der Helgoländer Bucht. Veröffentlichungen des Instituts für Meeresforschung in Bremerhaven, 12, 95-141.

Süßbach, S. \& Breckner, A., 1911. Die Seeigel, Seesterne und Schlangensterne der Nord- und Ostsee. Wissenschaftliche Meeresuntersuchungen. Kommission zur Wissenschaftlichen Untersuchung der Deutschen Meere in Kiel und der Biologischen Anstalt Helgoland, 12, 169-300.

Thiel, M. \& Gutow, L., 2005. The ecology of rafting in the marine environment - II. The rafting organisms and community. Oceanography and Marine Biology. Annual Review, 43, 279-418.

Thatje, S. \& Gerdes, D., 1997. The benthic macrofauna of the inner German Bight: Present and past. Archive of Fishery and Marine Research, 45, 93-111.

Unno, J., 2000. Occurrence of Amphipholis squamata (Echinodermata: Ophiuroidea) in relation to habitat in the Leschenault Inlet estuary. Fournal of the Royal Society of Western Australia, 83, 475-480.
Ursin, E., 1960. A quantitative investigation of the Echinoderm Fauna of the Central North Sea. Meddelelser Fra Danmarks Fiskeri-Og Havunders Øgelser, 2, 1-204.

Warner, G.F., 1971. On the ecology of a dense bed of the brittlestar Ophiothrix fragilis. Fournal of the Marine Biological Association of the United Kingdom, 51, 267-282.

Warner, G.F., 1982. Food and feeding mechanisms: Ophiuroidea. In Echinoderm nutrition (ed. M. Jangoux and J.M. Lawrence), pp. 161-181. Rotterdam: A.A. Balkema.

Webb, C.M. \& Tyler, P.A., 1985. Post-larval development of the common north-west European brittles stars Ophiura ophiura, $O$. albida and Acrocnida brachiata (Echinodermata: Ophiuroidea). Marine Biology, 89, 281-292.

Wiltshire, K.H. \& Manly, B.F.J., 2004. The warming trend at Helgoland Roads, North Sea: phytoplankton response. Helgoland Marine Research, 58, 269-273.

Ziegelmeier, E., 1978. Macrobenthos investigations in the eastern part of the German Bight from 1950 to 1974. Rapport et Procèsverbaux des Réunion. Conseil Permanent International pour l'Exploration de la Mer, 172, 432-444.

Submitted 16 September 2005. Accepted 13 April 2006. 